\title{
PERFIL DE MORTALIDADE MATERNA EM UMA MATERNIDADE PÚBLICA DA CIDADE DE MANAUS-AM
}

\author{
MATERNAL MORTALITY PROFILE IN A PUBLIC MATERNITY \\ HOSPITAL IN THE CITY OF MANAUS-AM
}

\section{Bárbara Misslane da Cruz Castro', Semírames Cartonilho de Souza Ramos ${ }^{2}$}

\section{RESUMO}

Objetivo: Analisar os casos de óbitos maternos ocorridos em uma maternidade pública de referência da cidade de Manaus. Metodologia: Estudo quantitativo, do tipo documental, com base na análise de 41 fichas de Investigação hospitalar e declarações de óbitos maternos ocorridos durante o ciclo gravídico-puerperal no período de janeiro de 2010 a dezembro de 2014. Os resultados foram analisados por meio do Software SAS for Windows ${ }^{\circledR}$. Resultados: Os dados permitiram a identificação do perfil dos óbitos maternos na instituição estudada, constituindo grupo de risco mulheres com idade entre 30-39 anos, pardas, solteiras, do lar, com baixo nível de escolaridade, residentes na zona norte de Manaus, primíparas e que realizaram de 1 a 3 consultas de pré-natal. Conclusão: A análise revela vulnerabilidades socioeconômicas e iniquidades, indica fragilidades nas políticas públicas e inadequação no acesso aos bens e serviços de saúde.

Descritores: Morte Materna; Saúde da Mulher; Perfil de Saúde.

\section{ABSTRACT}

Objective: To analyze the cases of maternal deaths which occured in a public maternity hospital of reference in Manaus. Methodology: Quantitative and documental study based in the analyze of 41 hospital investigation forms and maternal death certificates occured during the gravidic-puerperal cycle from January 2010 to December 2014. The results were analyzed using the Software SAS for Windows ${ }^{\circledR}$. Results: The data allow the identification of the profile of maternal deaths in the studied institution, constituting risk group women aged 30-39 years, brown, single, home with low level of education, residents in the north of Manaus, gilts and They held 1-3 prenatal consultations. Conclusion: The analysis reveals socioeconomic inequities and vulnerabilities, indicates weaknesses of public policies, inadequate access to health goods and services.
${ }^{1}$ Especialista em Residência em Enfermagem Obstétrica pela Universidade Federal do Amazonas (UFAM), Manaus, AM, Brasil.

${ }^{2}$ Doutora em Enfermagem pela Universidade Estadual de São Paulo (UNESP), São Paulo, SP, Brasil.

Descriptors: Maternal Death; Women's Health; Health Profile. 


\section{Introdução}

A morte materna é a morte de uma mulher durante a gestação ou dentro de um período de 42 dias após o término desta, independente da duração ou da localização da gravidez, devido a qualquer causa relacionada com a gestação. No entanto não é considerada morte materna a que é provocada por fatores acidentais ou incidentais ${ }^{1}$.

De acordo com o Ministério da Saúde as mortes maternas podem ser por causas obstétricas diretas ou indiretas. A primeira se refere às decorrentes de complicações obstétricas durante a gravidez, parto ou puerpério consequentes de intervenções, omissões, tratamento incorreto ou da ocorrência concomitante de qualquer uma dessas causas. Conceitualmente, a morte materna obstétrica indireta diz respeito àquela resultada de doenças pregressas a gestação ou que se desenvolveram neste período, não sendo provocadas por causas obstétricas diretas, mas agravadas pelos efeitos fisiológicos da gravidez¹ .

A Razão de Mortalidade Materna (RMM) é classificada pela Organização Mundial de Saúde (OMS) do seguinte modo: RMM baixa quando menor que 20 óbitos por 100.000 nascidos vivos (NV), RMM alta entre 50 e 149 óbitos e RMM muito alta a partir de 150 por 100.000 NV$^{2}$.

No ano 2000, o Brasil foi signatário de acordo internacional com a "Declaração do Milênio", onde foram estipulados oito objetivos para o desenvolvimento do milênio, sendo um deles a melhoria da saúde da mulher com e redução em $3 / 4$ dos índices de mortalidade materna entre os anos de 1990 a 2015³. A partir da década de 90 o Brasil passou por importantes avanços socioeconômicos inclusive na saúde, no entanto as taxas de mortalidade materna continuam elevadas, constituindo um nó crítico para a qualidade e impacto da política de humanização nos serviços que realizam pré-natal ${ }^{4}$.

Uma das metas do Brasil é reduzir até 2015 a RMM a valor igual ou inferior a 35 óbitos maternos por 100 mil nascidos vivos. Para atingir este objetivo, a redução anual média da RMM no Brasil deve ser de no mínimo 5,5\% ${ }^{5}$. Nesta perspectiva, monitorar esse indicador pode ser útil para apoiar a tomada de decisão, a focalização de grupos de maior risco, a identificação de hipóteses causais e definição da necessidade de estudos futuros ${ }^{6}$. No ano de 2010 a RMM do Brasil foi de 60 óbitos maternos para 100.000 NV, seguidos de 63 óbitos na região Norte, 94 no Estado do Amazonas e 84 óbitos no município de Manaus. Essas taxas revelam valores elevados da Razão de Mortalidade Materna?.

A mortalidade materna é um indicador sensível à qualidade de vida de uma população, trata-se de um indicador de análise das mortes precoces, evitáveis, que invariavelmente atingem as mulheres com menor acesso aos bens sociais ${ }^{3}$. A ocorrência de óbitos maternos reflete, ainda, a falta de qualidade da assistência prestada nos serviços de saúde, bem como a insatisfatória operacionalização das políticas públicas voltadas à saúde da mulher. Tal condição é objeto de grandes preocupações e discussões, uma vez que se trata de um evento presente com alta incidência nos países em desenvolvimento, atingindo principalmente as populações mais vulneráveis ${ }^{8}$.

No Brasil as principais causas de óbitos maternos registrados em 2010, foram por causas obstétricas diretas. Entre as causas com maior impacto na ocorrência destes óbitos estão a hipertensão (19,7\%), a hemorragia (10,9\%) a infecção puerperal $(6,5 \%)$ e o abortamento $(4,6 \%)$. Em relação aos óbitos por causas indiretas, as doenças do aparelho circulatório complicadas pela gestação, parto ou puerpério foram os eventos mais frequentes, repercutindo-se em $7,6 \%$ do total de óbitos 5 .

No Brasil a segunda área de maior prevalência de óbitos maternos é a região Norte e os locais de maior ocorrência são nas maternidades ${ }^{8}$. Frente ao exposto é possivel estimar a magnitude desses óbitos, sendo ação fundamental para revelar informações que contribuam para instrumentalizar ações referentes a vigilância sanitária, refletindo a necessidade de melhoria da qualidade dos serviços obstétricos, da assistência oferecida no município de Manaus/AM e da inserção de tais discussões em espaços de arguição da comunidade, fortalecendo o controle social da saúde.

Nesse sentido, o objetivo da pesquisa foi analisar os casos de óbitos maternos ocorridos em uma maternidade pública de referência da cidade de Manaus.

\section{Metodologia}

A pesquisa caracteriza-se por um estudo quantitativo, exploratório, retrospectivo, do tipo documental (dados secundários) com o objetivo de avaliar eventos que ocorreram no passado e quantificá-los, nesse caso, avaliar casos de óbitos maternos ocorridos em uma maternidade pública estadual do município de Manaus, através da análise de fichas de Investigação hospitalar, declarações de óbitos maternos e prontuários. 
A escolha da instituição justifica-se pelo fato de apresentar um dos serviços com maior ocorrência de óbitos maternos no estado do Amazonas, considerando o período de 2010, 2011 e 2012 de acordo com os dados da Rede Cegonha - Ações Estratégicas para a redução da Morte Materna9.

Foram selecionadas para análise 41 fichas de investigação hospitalar, declarações de óbitos e prontuários de casos de óbitos maternos de mulheres com idade entre 10 a 49 anos ocorridos durante a gravidez, parto ou puerpério no período de janeiro de 2010 a dezembro de 2014. Foram excluídas deste estudo fichas de investigação hospitalar de óbitos de mulheres em idade fértil não atribuídos a causas obstétricas, assim como fichas de investigação ilegíveis, de acesso impossibilitado por estarem sob júdice, as cujo limite temporal extrapola os estabelecidos por este estudo e as de óbitos maternos tardios.

Das declarações de óbitos e fichas de investigação hospitalar de óbito materno foram extraídas, através de um instrumento de coleta de dados elaborado pelas autoras, informações sociodemográficas, epidemiológicas e obstétricas, como: idade, naturalidade, município de residência, zona de bairro, estado civil, raça/cor, escolaridade, profissão/ ocupação, ano do óbito, realização de pré-natal de risco habitual e de alto risco, se gestante de alto risco, local de ocorrência do óbito, ocorrência do óbito (em que momento), ocorrência do óbito após aborto; ocorrência do óbito durante parto ou puerpério, classificação do óbito (obstétrico direto ou indireto) e paridade.

Os dados obtidos foram registrados no programa $E x c e^{\circledR}$, e os cálculos estatísticos foram realizados pelo Software SAS for Windows ${ }^{\circledR}$, versão 9.2, sendo utilizada a análise estatística descritiva, com porcentagem simples.

Para a realização e elaboração da pesquisa foram consideradas as orientações da Resolução No 466/2012 do Conselho Nacional de Saúde, após aprovação do projeto pelo Comitê de Ética em Pesquisa da Universidade Federal do Amazonas (UFAM) sob o registro CAAE: 36318914.6.0000.502.

\section{Resultados}

Na Maternidade Balbina Mestrinho no período correspondente a janeiro de 2010 a dezembro de 2014, ocorreram 41 óbitos maternos por causas obstétricas de mulheres em idade fértil, compreendidos entre a faixa etária de 10 a 49 anos. Sendo 07 (17\%) óbitos ocorridos em 2010, 05 (12\%) em 2011, 07 (17\%) em 2012, 16 (39\%) em 2013 e 06 (15\%) em 2014.

A tabela 1 demonstra a distribuição de óbitos maternos segundo dados sócio-demográficos: faixa etária, naturalidade, município de residência, zona de bairro, profissão/ocupação, escolaridade, estado civil e raça/cor.

Tabela 1 - Distribuição de óbitos maternos segundo dados sócio-demográficos. Manaus, AM, Brasil 2010-2014.

\begin{tabular}{c|c|c}
\hline Idade & N & $\%$ \\
\hline 10-14 anos & 0 & 0 \\
\hline 15-19 anos & 12 & 29 \\
\hline 20- 29 anos & 10 & 24 \\
\hline 30-39 anos & 17 & 41 \\
\hline 40-49 anos & 2 & 6 \\
\hline Naturalidade & 25 & 60 \\
\hline Manaus & 16 & 40 \\
\hline Outros Municípios do Amazonas & \multicolumn{2}{|c}{} \\
\hline Município de Residência & 34 & 83 \\
\hline Manaus & 7 & 17 \\
\hline \multicolumn{2}{c|}{ Outros Municípios do Amazonas }
\end{tabular}




\begin{tabular}{|c|c|c|}
\hline Zona de Bairro & $\mathbf{N}$ & $\%$ \\
\hline Norte & 10 & 24 \\
\hline Sul & 8 & 20 \\
\hline Leste & 7 & 17 \\
\hline Oeste & 3 & 7 \\
\hline Centro- Sul & 3 & 7 \\
\hline Centro-Oeste & 4 & 10 \\
\hline Outros & 6 & 15 \\
\hline \multicolumn{3}{|l|}{ Profissão/ocupação } \\
\hline Do Lar & 18 & 44 \\
\hline Diarista & 2 & 5 \\
\hline Estudante & 11 & 27 \\
\hline Professora & 1 & 2 \\
\hline Desempregada & 2 & 5 \\
\hline Outras & 7 & 17 \\
\hline \multicolumn{3}{|l|}{ Escolaridade } \\
\hline Zero & 2 & 5 \\
\hline 1 a 3 anos & 18 & 44 \\
\hline 4 a 7 anos & 8 & 19 \\
\hline 8 a 11 anos & 7 & 17 \\
\hline 12 anos ou mais & 2 & 5 \\
\hline Sem informação & 4 & 10 \\
\hline \multicolumn{3}{|l|}{ Estado Civil } \\
\hline Solteira & 28 & 68 \\
\hline Casada & 4 & 10 \\
\hline União Estável & 8 & 20 \\
\hline Não Informado & 1 & 2 \\
\hline \multicolumn{3}{|l|}{ Raça/Cor } \\
\hline Pardas & 33 & 80 \\
\hline Brancas & 6 & 15 \\
\hline Indígenas & 2 & 5 \\
\hline Negra & 0 & $\mathrm{O}$ \\
\hline Total & 41 & 100 \\
\hline
\end{tabular}

A maioria dos casos de óbitos maternos ocorreu em mulheres com idade entre 30-39 anos (41\%), naturais do município de Manaus (60\%), residentes no município de Manaus (83\%), na zona Norte (24\%), do lar (44\%), com baixa escolaridade $(44 \%)$, sem cônjuge $(68 \%)$, de raça/cor pardas $(60 \%)$.

Em relação às variáveis obstétricas, na tabela 2 estão distribuídos os óbitos maternos segundo paridade, consultas de pré-natal, risco gestacional, pré-natal de alto risco e momento da ocorrência do óbito. 
Tabela 2 - Distribuição de óbitos maternos segundo paridade, consultas de pré-natal, risco gestacional, pré-natal de alto risco e momento do óbito. Manaus, Am, Brasil, 2010-2014.

\begin{tabular}{|c|c|c|}
\hline Paridade & $\mathbf{N}$ & $\%$ \\
\hline $\mathrm{Gl}$ & 17 & 41 \\
\hline $\mathrm{G} 2$ & 11 & 27 \\
\hline G3 ou mais & 13 & 32 \\
\hline \multicolumn{3}{|l|}{ Consultas } \\
\hline 0 & 5 & 12 \\
\hline De 1-3 & 10 & 24 \\
\hline De 4-6 & 8 & 20 \\
\hline$>6$ & 2 & 5 \\
\hline Não informado & 16 & 39 \\
\hline \multicolumn{3}{|l|}{ Risco Gestacional } \\
\hline Alto Risco & 13 & 32 \\
\hline Baixo Risco & 28 & 68 \\
\hline \multicolumn{3}{|l|}{ Pré-natal de alto risco } \\
\hline Realizou pré-natal de alto risco & 3 & 7 \\
\hline \multicolumn{3}{|l|}{ Momento do óbito } \\
\hline Puérperio & 25 & 62 \\
\hline Após o abortamento & 5 & 12 \\
\hline Durante a gestação & 10 & 24 \\
\hline No parto ou até 1 hora após & 1 & 2 \\
\hline Total & 41 & 100 \\
\hline
\end{tabular}

A maior parte dos óbitos ocorreu em primíparas (41\%), que realizaram de 1 a 3 consultas de pré-natal (24\%). Observa-se que das mulheres que foram a óbito, $32 \%$ eram gestantes de alto risco, sendo que apenas $7 \%$ realizaram pré-natal de alto risco. Quando considerado o momento de ocorrência do óbito, $62 \%$ foram no puerpério.

Tabela 3 - Distribuição de óbitos maternos por causas obstétricas diretas e indiretas. Manaus, Am, Brasil, 2010-2014.

\begin{tabular}{c|c|c}
\hline Obstétricas Indiretas & $\mathbf{N}$ & $\%$ \\
\hline Sepse & 2 & 5 \\
\hline H1N1 & 3 & 7 \\
\hline Insuficiência de Múltiplos órgãos & 4 & 10 \\
\hline Choque Cardiogênico + Cardiopatia & 1 & 2 \\
\hline Doença Mista do Tecido Conjuntivo & 1 & 2 \\
\hline Choque Séptico & 7 & 17 \\
\hline Insuficiência Respiratória Aguda & 1 & 2 \\
\hline AIDS & 1 & 2 \\
\hline Insuficiência Renal Aguda & 1 & 2 \\
\hline Varicela & 1 & 2 \\
\hline AVC hemorrágico & 1 & $\mathbf{5 6}$ \\
\hline
\end{tabular}




\begin{tabular}{c|c|c}
\hline Obstétricas Diretas & N & \% \\
\hline DHEG+ Síndrome de HELLP & 5 & 12 \\
\hline Infecção Puerperal & 3 & 7 \\
\hline Hemorragia & 8 & 20 \\
\hline Abortamento & 2 & 5 \\
\hline Total & $\mathbf{1 8}$ & $\mathbf{4 4}$ \\
\hline
\end{tabular}

Em relação às causas de óbitos maternos observa-se na tabela 3 a distribuição de mortes por causas obstétricas diretas e indiretas.

As causas predominantes de óbitos foram às indiretas (56\%), com destaque para o Coque Séptico (17\%), seguido de Insuficiência de Múltiplos órgãos (10\%) e H1N1 (7\%). As causas obstétricas indiretas representam 44\% de mortes, sendo a primeira causa por hemorragia (20\%), seguida de DHEG+Síndrome de Hellp (12\%) e Infecção Puerperal $(7 \%)$

\section{Discussão}

\section{Características sócio-demográficas}

Neste estudo a ocorrência de óbitos maternos foi predominante na faixa etária de 30-39 anos (41\%). Prevalência também observada em estudos cujo percentual representa $46 \%$ de óbitos maternos em idade de 30-39 anos ${ }^{10,11}$. Tal achado corrobora com a literatura, uma vez que o maior risco de morte materna se encontra em mulheres jovens ou com idades mais avançadas (menores de 15 anos ou maiores de 35 anos) sendo as mais velhas as que apresentam maior suscetibilidade a doença hipertensiva específica da gestação. Considera-se que a idade materna menor que 17 e maior que 35 anos representa um fator de risco importante na gravide $z^{5}$.

Apesar da idade ser uma importante variável de análise deste estudo é importante a inferência de que a faixa etária não pode ser considerada um fator isolado para a ocorrência de complicações maternas e fetais, outras variáveis como condições de vida e saúde das gestantes e a qualidade da assistência à saúde reprodutiva, ao pré-natal, parto e pós-parto são de grande significância para os desfechos perinatais ${ }^{5}$.

Em relação à procedência das mulheres, identificou-se que 83\% residiam em Manaus, onde há 08 maternidades públicas, sendo 02 de referência obstétrica de alta complexidade, com cobertura da Estratégia Saúde da Família (ESF) é de $35 \%$ e do pré-natal de 4 a 6 consultas $(41,97 \%)$ e 7 ou mais $(39,96 \%)^{6}$. Diante destas informações infere-se que a ocorrência destes óbitos pode estar atrelada a baixa cobertura e qualidade do pré-natal, despreparo profissional, inadequação da assistência no atendimento às urgências e emergências obstétricas e perinatais ou omissões no tratamento.

A procedência de mulheres de outros municípios do estado do Amazonas (17\%) pode ser indicativo da inexistência de uma rede serviços obstétricos nas regiões de origem desta população, uma vez que há uma peregrinação pela assistência em uma rede de serviços fragmentada e não resolutiva, característica tanto da capital quanto das periferias/interior do estado.

Frente ao exposto há indícios de busca pela assistência no anteparto em serviços dos grandes centros urbanos, condição bastante comum na realidade brasileira, repercutindo em demora no atendimento obstétrico com consequentes tragédias maternas e neonatais.

Ao analisar a distribuição dos óbitos maternos em relação à profissão/ocupação, foi observado que $68 \%$ das mulheres eram do lar, atividade por vezes desvalorizada por não ser remunerada e relacionada ao desgaste físico das mulheres, considerando-se um risco para a ocorrência do óbito ${ }^{11}$.

Em relação ao nível de escolaridade, $44 \%$ das mulheres possuíam o ensino fundamental incompleto ( 1 a 3 anos de estudo). Sabe-se que a baixa escolaridade pode afetar negativamente a formulação de conceitos de autocuidado em saúde, a noção de conservação ambiental e a percepção da necessidade de atuação do indivíduo como cidadão em contextos sanitários coletivos ${ }^{12}$.

Outro achado deste estudo é a ocorrência de óbitos em maior proporção nas mulheres solteiras (68\%), corroborando com vários estudos em capitais brasileiras ${ }^{13,14}$. A presença de um companheiro é considerada importante durante o período gestacional, sendo compreendida como possibilidade de aumento do suporte emocional e financeiro 
à mulher, fatores condicionantes para o desenvolvimento de complicações maternas. Deste modo é possível inferir-se que gestantes solteiras, assim como as viúvas e separadas judicialmente, constituem um grupo vulnerável, uma vez que na grande maioria dos casos há ausência de aporte afetivo, emocional, social, financeiro e de estímulo ao autocuidado.

Outra variável analisada foi a raça das mulheres que evoluíram a óbito, sendo as pardas $80 \%$ desta população, condição que converge com estes eventos na maioria das regiões brasileiras embora os percentuais variem de acordo com a composição de cor/raça predominante localmente $e^{4,13,15,16}$. Em contrapartida a este achado, um estudo evidencia que em várias capitais brasileiras as mulheres negras apresentaram maiores Razões de Mortalidade Materna, e quando comparadas as brancas apresentam maiores riscos de doenças hipertensivas e crônicas ${ }^{17}$.

Outro estudo que traçou o perfil epidemiológico das mortes maternas em Itajaí no período de 1997-2007 identificou que $88 \%$ dessas mortes foram atribuídas a mulheres da raça branca, e apenas $12 \%$ em negras, não havendo casos de mulheres amarelas ${ }^{10}$. Frente a tal análise torna-se impreciso apontar a cor que apresenta maior risco em decorrência da intensa miscigenação racial.

Em consonância aos achados deste estudo, observou-se em outros estudos a predominância de óbitos em mulheres pardas (78,59\%), donas de casa $(52,48 \%)$ e solteiras $(66,06 \%) .16$ Esta evidência pode estar relacionada às similaridades regionais e culturais entre Manaus e o Estado do Pará.

\section{Características obstétricas}

Em relação à paridade, identificou-se que $41 \%$ das mulheres eram primíparas. Estudos relativos à paridade são controversos $^{15,18}$. Há estudo que aponta que a influência da paridade sobre as mortes maternas predomina nas nulíparas sobre as demais, de forma significativa ${ }^{18}$.

Em relação à assistência pré-natal, percebeu-se uma deficiência no registro dessa variável, o correspondente a 39\% dos casos. Trata-se de um fato preocupante, uma vez que dificulta o reconhecimento dos níveis de atenção à saúde que apresentam maiores deficiências na assistência à saúde da mulher gestante. Identificou-se que $24 \%$ das mulheres realizaram de 1 até 3 consultas. Diferença significativa é observada em um estudo ${ }^{19}$, das 37 mulheres que vieram a óbito, $19(51,3 \%)$ haviam realizado mais de seis consultas, seguido por 10 (27\%) com menos de três e $6(16,2 \%)$ entre três e cinco consultas.

Em relação ao risco gestacional, observou-se que $32 \%$ das gestantes eram de alto risco e apenas $7 \%$ realizaram acompanhamento de pré-natal de alto risco. Classifica-se como gestantes de alto risco, o grupo de mulheres portadoras de alguma doença ou que sofreram algum agravo ou desenvolveram problemas de saúde durante a gravidez, com probabilidade de evolução desfavorável tanto para a mãe quanto para o bebê ${ }^{20}$.

No presente estudo, identificou-se que a maior parte dos óbitos ocorreu no período puerperal até 41 dias após 0 parto, correspondendo a $61 \%$ dos casos. Apesar da ocorrência de maior número de complicações no puerpério serem responsáveis pelo aumento nos casos de óbitos maternos, outras causas de óbitos estão também ligadas às complicações ocorridas na gestação ou parto e abortamento. Para evitá-las, é essencial a atuação de uma equipe capacitada, que reconheça as complicações de cada fase do ciclo gravídico puerperal e o momento adequado das intervenções ${ }^{5}$.

Esse resultado também tem associação com os achados dos estudos realizados em Fortaleza e São Luiz, que tiveram como período de ocorrência da maioria dos óbitos "no momento do parto" e "do pós-parto imediato", apontando que esses momentos críticos devem ser adequadamente assistidos pela equipe de saúde, incluindo a presença de especialistas na assistência ao trabalho de parto, fato este que poderia assegurar melhor qualidade no atendimento à mulher, podendo evitar a morte materna ${ }^{21,23}$.

Para a detecção de intercorrências próprias do período puerperal, é imprescindível pelo menos uma visita ambulatorial, em torno do sétimo dia após o parto, com realização de uma anamnese detalhada e exame físico completo da puérpera, para diagnosticar sinais de anemia e/ou infecção. A segunda consulta deverá ser feita em 30 a 42 dias após o parto, para reavaliação da saúde materna e orientações relativas aos métodos contraceptivos ${ }^{20}$.

Quanto ao local de ocorrência dos óbitos, 95\% ocorreram na Maternidade Balbina Mestrinho. Outros autores também observaram que as mortes maternas em sua maioria ocorreram em ambiente hospitalar ${ }^{4,14,16,21}$.

Dentre os óbitos maternos obstétricos diretos, 20\% foram por hemorragia, 12\% por DHEG+Síndrome de HELLP, $7 \%$ por infecção puerperal e $5 \%$ por abortamento, totalizando $44 \%$ dos óbitos. Nos obstétricos indiretos as principais causas foram por Choque Séptico 17\%, Insuficiência de Múltiplos órgãos 10\%, 7\% por H1N1, seguidos por outras causas, totalizando $56 \%$. 
Observou-se relação inversa entre a mortalidade materna por causas obstétricas diretas e indiretas em relação ao panorama atual brasileiro. Visto que neste estudo houve o predomínio atípico de mortes maternas por causas obstétricas indiretas, correspondendo a 56\% dos casos. Esse comportamento foi observado em um estudo de 2006 sobre a análise do perfil da mortalidade materna no Hospital das Clínicas da Faculdade de medicina de Ribeirão Preto onde a predominância de óbitos maternos por causas obstétricas indiretas correspondeu a 47,8\% dos casos. Resultado este não encontrado em outros referenciais com período superior ao ano de $2006^{22}$.

Estudo realizado em Manaus nos anos de 2001 a 2010, identificou a predominância de 67,2\% de MM por causas obstétricas diretas e $25,7 \%$ de indiretas. As principais causas de MM foram infecção puerperal, hipertensão arterial, hemorragia e abortamento, causas diretas e potencialmente evitáveis. Entre as indiretas, a maioria das mortes ocorreu por doenças cardíacas e respiratórias ${ }^{23}$.

Outro estudo identificou que na América Latina e em países do Caribe a proporção de mortes foi de 3,8\% para causas diretas e 3,9\% para causas indiretas ${ }^{24}$. Países onde a RMM é aceitável conforme classificação da OMS predominaram as causas de mortes maternas indiretas ${ }^{25}$.

\section{Considerações Finais}

Os resultados deste estudo evidenciaram que no período de janeiro de 2010 a dezembro de 2014 a maior proporção de mortes maternas ocorreu em mulheres no auge da vida reprodutiva, com idade entre 30-39 anos, de raça/cor parda, solteiras, do lar, com baixo nível de escolaridade, naturais de Manaus, residentes na zona norte da cidade. Com relação às variáveis obstétricas identificou-se que a maioria das mulheres eram primíparas, que realizaram de 1 a 3 consultas de prénatal, cuja evolução do óbito deu-se durante o período puerperal, no estabelecimento de saúde. Houve predominância de óbitos por causas obstétricas indiretas, causa que diverge de alguns estudos na maioria dos estados brasileiros.

Embora as causas obstétricas diretas contribuam com incidência elevada de óbitos maternos no Brasil, as mesmas são consideradas evitáveis, passíveis de prevenção. Já as obstétricas indiretas são de menor ocorrência e difíceis de serem controladas, uma vez, que são decorrentes de patologias graves associadas à gestação sendo muitas vezes inevitáveis.

A maternidade do estudo por ser um centro obstétrico de referência na atenção ao pré-natal de alto risco, consequentemente concentra a maior parte dos óbitos por causas indiretas.

Ao longo da realização deste estudo percebeu-se o preenchimento inadequado das declarações de óbitos e fichas de investigação de óbito materno. A falta de informações relevantes para esta pesquisa comprometeu em partes uma avaliação fidedigna dos itens estudados. É necessário conscientizar os profissionais envolvidos no processo do preenchimento dos dados sobre a importância epidemiológica da mortalidade materna para os serviços de saúde bem como para a sociedade.

Evidentemente, a discussão sobre morte materna não se restringe a este estudo, uma vez que é necessário aprofundar e analisar de forma mais ampliada os fatores de risco associados ao óbito materno, devido à complexidade do tema e o vislumbramento de muitas possibilidades de investigação quanto à mortalidade materna na cidade de Manaus.

Espera-se que este estudo seja de utilidade em pesquisas futuras que tenham como enfoque o estudo sobre 0 perfil sociodemográfico e obstétrico de óbitos maternos no município de Manaus, incentivando os profissionais de saúde envolvidos no cuidado as gestantes e mulheres a reverem práticas e aprimorarem a assistência.

\section{Referências}

1. Ministério da Saúde. Guia de vigilância epidemiológica do óbito materno. Brasília (Brasil): Ministério da Saúde, 2009.

2. Brasil. Mato Grosso. Secretaria de Estado de Saúde. Manual de indicadores e parâmetros da saúde. Cuiabá: Secretaria de Estado de Saúde, 2010.

3. Organização Mundial de Saúde. CID-10: Classificação Estatística Internacional de Doenças e Problemas Relacionados à Saúde. Décima Revisão (CID-10). 8.a ed. São Paulo: Edusp, 2010.

4. Souza MS, Garção GS, Barreto SMSS, Menezes MO, Silva DP, Souza CS. et al. Mortalidade Materna: Perfil Epidemiológico em Sergipe (2001-2010). Cadernos de Graduação - Ciências Biológicas e da Saúde. [Internet]. 2013 [acesso em: 21 de out 2015]; 1(17): 49-58. Disponível em: http://periodicos.set.edu.br/index.php/cadernobiologicas/ article/view/1009/535. 5. Ministério da Saúde. Mortalidade materna no Brasil: principais causas de morte e tendências temporais no período de 1990 a 2010. Brasília (Brasil): Ministério da Saúde, 2011. 
6. Barbastefano OS, Vargens OMC. Prevenção da mortalidade materna: desafio para o enfermeiro. Rev. bras. enferm. [Internet]. 2009 [acesso em: 18 de jan 2015]; 62(2): 278-82. Disponível em: http:/www.scielo.br/pdf/reben/v62n2/a17v62n2.pdf.

7. DATASUS [Internet]. Brasília: Ministério da Saúde (BR) [acesso em: 12 de jul 2015]. Departamento de Informática do SUS - DATASUS. Painel de Monitoramento de Mortalidade Materna. Disponível em: http://www.datasus.gov.br.

8. Chrestani MA, Santos IS, Cesar JÁ, Winckler LS, Gonçalves TS, Neumann NA. Assistência à gestação e ao parto: resultados de dois estudos transversais em áreas pobres das regiões Norte e Nordeste do Brasil. Cad. de Saúde Pública. [Internet]. 2008 [acesso em: 20 de mai 2015]; 24(7): 1609 -1618. Disponível em: http://www.scielo.br/pdf/csp/v24n7/16.pdf.

9. Ministério da Saúde [Internet]. Rede Cegonha - Ações Estratégicas para a redução da Morte Materna. Brasília: Ministério da Saúde, 2012. [acesso em: 19 de Mai 2015]. Disponível em: http://portal.saude.gov.br/portal/arquivos/pdf/ rede_cegonha_afssss.pdf.

10. Lavado MM, Poener CA, Tomaz TSO, Morelli C, Ramos LR, Koch TF. et al. Perfil epidemiológico das mortes maternas em Itajaí no período de 1997-2007. Arq. Catarin. Med. [Internet]. 2012 [acesso em: 07 de mai 2015]; 41 (1): 28-33. Disponível em: http://www.acm.org.br/revista/pdf/artigos/908.pdf.

11. Riquinho DL, Correia SG. Mortalidade materna: perfil sócio-demográfico e causal. Rev Bras Enferm. [Internet] 2006 [acesso em: 09 de abr 2015]; 59(3): 303-7. Disponível em: http://www.scielo.br/pdf/reben/v59n3/a10v59n3.pdf.

12. Keffler K, Souza SRRK, Wall ML, Martins M, Moreira SDR. Características sociodemográficas e mortalidade materna em um hospital de referência na cidade de Curitiba - Paraná. Cogitare Enferm. [Internet] 2010 [acesso em: 09 de jan 2015]; 15 (3): 500-5. Disponível em: http://ojs.c3sl.ufpr.br/ojs/index.php/cogitare/article/viewArticle/18894.

13. Buna CMSC, Sampaio SLP, Sousa MEL, Fonseca LMB, Caldas AJMC, Aquino DMC. Caracterização da mortalidade materna ocorrida no município de São Luís no período de 2001- 2011. Rev Pesq Saúde. [Internet] 2014 [acesso em: 01 de jul 2015]; 15 (2): 296-300. Disponível em: http://www.periodicoseletronicos.ufma.br/index.php/revistahuufma/article/view/3268. 14. Brayner MC. Perfil dos óbitos maternos tardios de mulheres residentes no estado de Pernambuco, Quadriênio 2005-2008. [monografia]. 2011 [acesso em: 26 de jun 2015]. Disponível em: http://www.cpqam.fiocruz.br/bibpdf/2011brayner-mc.pdf.

15. Ferraz L, Bordignon M. Mortalidade materna no Brasil: uma realidade que precisa melhorar. Rev. Baiana de Saúde Pública. [Internet] 2012 [acesso em: 13 de abr 2015]; 36 (2): 527-538. Disponível em: http://inseer.ibict.br/rbsp/index.php/ rbsp/article/viewFile/474/pdf_150.

16. Botelho NM, Silva IFMM, Tavares JR, Lima LO. Morte materna no estado do Pará: aspectos epidemiológicos. Rev. para. med. [Internet] 2013 [acesso em: 22 de dez 2014]; 27 (1). Disponível em: http://files.bvs.br/upload/S/0101-5907/2013/v27n1/a3503.pdf.

17. Martins AL. Mortalidade materna de mulheres negras no Brasil. Cad Saúde Pública.[Internet] 2006 [acesso em: 25 de fev 2015]; 22 (11): 2473-9. Disponível em: http://www.scielo.br/scielo.php?script=sci_arttext\&pid=S0102$311 \times 2006001100022$.

18. Andrade ATL, Guerra MO, Andrade GN, Araújo DAC, Souza JP. Mortalidade materna: 75 anos de observações em uma maternidade escola. Rev. Bras. Ginecol. Obstet. [Internet] 2006 [acesso em: 23 de fev 2015]; 28 (7): 380-387. Disponível em: http://dx.doi.org/10.1590/S0100-72032006000700002.

19. Herculano MMS, Veloso LS, Teles LMR, Oriá MOB, AlmeidaPC, DamascenoAKC. Óbitos maternos em uma Maternidade Pública de Fortaleza: um estudo epidemiológico. Rev. esc. enferm. USP. [Internet] 2012 [acesso em: 27 de mai 2015]; 46 (2): 295-301. Disponível em: http://www.scielo.br/scielo.php?script=sci_arttext\&pid=S0080-62342012000200005.

20. Brasil . Ministério da Saúde. Atenção ao pré-natal de baixo risco. [Internet]. Brasília: Editora do Ministério da Saúde, 2012 [acesso em: 02 de fev 2015]. Disponível em: http://bvsms.saude.gov.br/bvs/publicacoes/cadernos_atencao_ basica_32_prenatal.pdf

21. Áfio ACE, Araújo MAL, Rocha AFB, Andrade RFV, Melo SP. Óbitos maternos: necessidade de repensar estratégias de enfrentamento. Rev Rene. [Internet] 2014 [acesso em: 03 de mar 2015]; 15 (4): 631-8. Disponível em: http://www. revistarene.ufc.br/revista/index.php/revista/article/view/1741/pdf39.

22. Gadelha OS, Costa AG, Mauad Filho F. Mortalidade materna no Hospital das Clínicas da Faculdade de Medicina de Ribeirão Preto, da Universidade de São Paulo. Revista da AMRIGS. [Internet] 2006 [acesso em 09 de fev 2015]; 50 (2): 135-138. Disponível em: http://www.amrigs.com.br/revista/50-2/ao04.pdf.

23. Ramos SCS, Calderon IMP. Análise de casos de mortes maternas em Manaus nos anos de 2001 a 2010 [tese]. Botucatu: Faculdade de Medicina da Universidade Estadual Paulista/ UNESP, 2013. 94 p. 24. González RP. Salud materno-infantil en las américas. Rev Chil Obstet Ginecol. [Internet] 2010 [acesso em 03 de fev 2015]; 75 (6): 411-421. Disponível em: http://dx.doi.org/10.4067/S0717 75262010000600011. 
25. Mello-Jorge MHP, Laurenti R, Gottlieb SLD. Análise da qualidade das estatísticas vitais brasileiras: a experiência de implantação do SIM e do Sinasc. Cienc Saude Coletiva. [Internet] 2007 [acesso em 27 de jul 2015]; 12(3): 643-54. Disponível: http://www.scielo.br/scielo.php?script=sci_pdf\&pid=\$141381232007000300014\&lng=en\&nrm=iso\&tlng=pt.

\section{Bárbara Misslane da Cruz Castro}

Endereço para correspondência - Conjunto Castelo Branco, Rua: 24, Bairro: Parque 10 de novembro, CEP: 69055-420, Manaus, AM, Brasil.

E-mail: barbaramisslane@hotmail.com

Lattes: http://lattes.cnpq.br/6311167485837370

Semírames Cartonilho de Souza Ramos - semiramesramosmoga@gmail.com

\section{Enviado em 05 de janeiro de 2016. Aceito em 01 de abril de 2016.}

